\title{
Oxaliplatin beim Kolorektalkarzinom: Von der Palliation zur Kuration
}

Mit jährlich fast 60000 Neuerkrankungen in Deutschland ist das kolorektale Karzinom eine der häufigsten Neoplasien. Zwar kann bei etwa $70 \%$ der betroffenen Patienten eine potenziell kurative Tumorresektion durchgeführt werden. Bei etwa der Hälfte der Patienten rezidiviert die Erkrankung jedoch und führt letztlich zum Tod. Doch kann eine adjuvante Chemotherapie auf Basis von 5-Fluorouracil (5-FU) die Prognose dieser Patienten deutlich verbessern: Im Vergleich zur alleinigen Resektion wird eine Mortalitätsreduktion um rund ein Drittel erreicht. Seit 1990 gilt daher die 6-monatige Therapie mit 5-FU/Folinsäure (5-FU/FS) im Stadium UICC III als Standard [20]. Bislang noch kontrovers diskutiert wird die adjuvante Chemotherapie für Patienten im Stadium II, da der Überlebensvorteil hier nur moderat ist. Daher beschränkt man die adjuvante Therapie in diesem Stadium meist auf Patienten mit erhöhtem Rezidivrisiko.

\section{FOLFOX4 auf dem Weg in die adjuvante Therapie}

Erst 2003 wurde auf der Jahrestagung der American Society of Clinical Oncology (ASCO) mit MOSAIC (Multicenter International Study of Oxaliplatin/5-FU/LV in the Adjuvant Treatment of Colon Cancer) eine adjuvante Therapiestudie vorgestellt, die die klinische Routine verändern wird: Die gerade im New England Journal of Medicine publizierte Studie [1] untersuchte die zusätzliche Gabe von Oxaliplatin $\left(\right.$ Eloxatin $^{\circledR}$ ) zu einem 5-FU/FS-Infusionsregime (FOLFOX4) - ein Protokoll, das sich bereits in der First-line-Therapie beim metastasierten Kolorektalkarzinom aufgrund der Steigerung von Ansprechraten und Überleben bewährt hat. In der multizentrischen MOSAIC-Studie wurden 2246 Patienten mit Kolonkarzinomen der Stadien II (T3 oder T4, N0, M0) und III (jedes T, N1 oder N2, M0) nach R0-Resektion randomisiert entweder dem Protokoll nach de Gramont (200 mg FS über 2 Std. i.v., $400 \mathrm{mg} / \mathrm{m}^{2}$ 5-FU als Bolus, $600 \mathrm{mg} / \mathrm{m}^{2}$ 5-FU i.v. über
22 Std., Tag 1 und 2) oder dem gleichen Schema plus Oxaliplatin $\left(85 \mathrm{mg} / \mathrm{m}^{2}\right.$ an Tag 1) zugeteilt [1]. Die Therapie wurde 14-tägig über insgesamt 12 Zyklen verabreicht. Im FOLFOX4-Arm erhielten 74,7\%, im Kontrollarm 86,5\% der Patienten alle geplanten Therapiezyklen. In beiden Gruppen konnten über $80 \%$ der geplanten Dosis appliziert werden. Die Patientencharakteristika beider Studienarme waren ausgewogen: Jeweils $60 \%$ der Teilnehmer hatten ein Kolonkarzinom im Stadium III, je 40\% einen Tumor im Stadium II.

\section{Zusätzliche Oxaliplatin-Gabe verhindert ein Viertel aller Rezidive}

Nach einem medianen Follow-up von knapp 38 Monaten waren im Kontrollarm 293 Rezidive und Todesfälle (26,1\%) aufgetreten, im FOLFOX4-Arm dagegen nur 237 Ereignisse $(21,1 \%)$. Daraus berechnet sich eine Hazard Ratio für Rezidive von 0,77 für den Oxaliplatin-haltigen Arm im Vergleich zur Kontrolle $(p=0,002)$. Anders ausgedrückt: In der MOSAICStudie konnte das Rezidivrisiko durch die zusätzliche Gabe von Oxaliplatin zur Standardtherapie mit 5-FU/FS um 23\% verringert werden (Abb. 1). Die Rate des krankheitsfreien Überlebens als primärem Endpunkt wurde von 72,9\% im Kontrollarm um absolut 5,3\% auf 78,2\% im FOLFOX4-Arm erhöht. Da die meisten Rezidive innerhalb der ersten 3 Jahre nach kurativer Therapie auftreten, gehen die Autoren jedoch davon aus, dass die Verbesserung des krankheitsfreien Überlebens auch in einer signifikanten Überlebensverlängerung resultieren wird.

\section{Effektivitätssteigerung nicht auf Kosten der Sicherheit}

Die zusätzliche Gabe von Oxaliplatin zum 5-FU/FS-Regime wurde generell gut toleriert. Häufigste Nebenwirkungen vom 


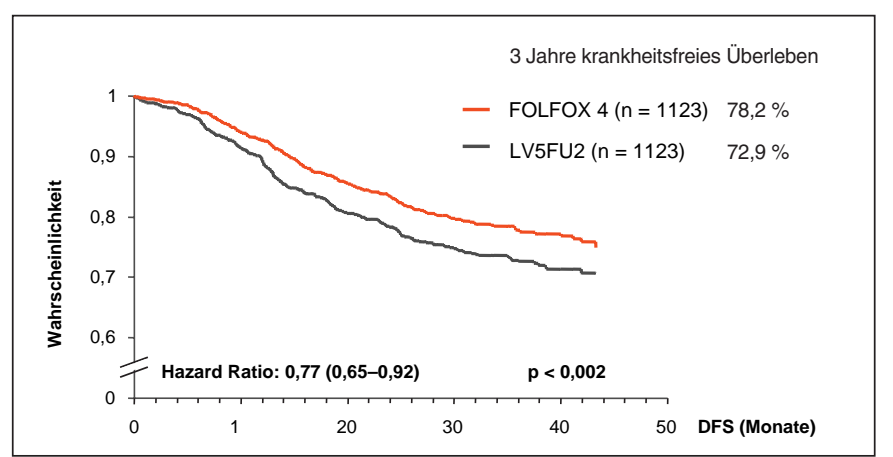

Abb. 1. MOSAIC-Studie: Signifikante Reduktion der Ereignisrate um 23\% nach 3 Jahren durch zusätzliche Gabe von Oxaliplatin zur 5-FU/FSTherapie [1]. DFS = Krankheitsfreies Überleben. FOLFOX4-Arm: 23\% Risikoreduktion gegenüber LV5FU2.

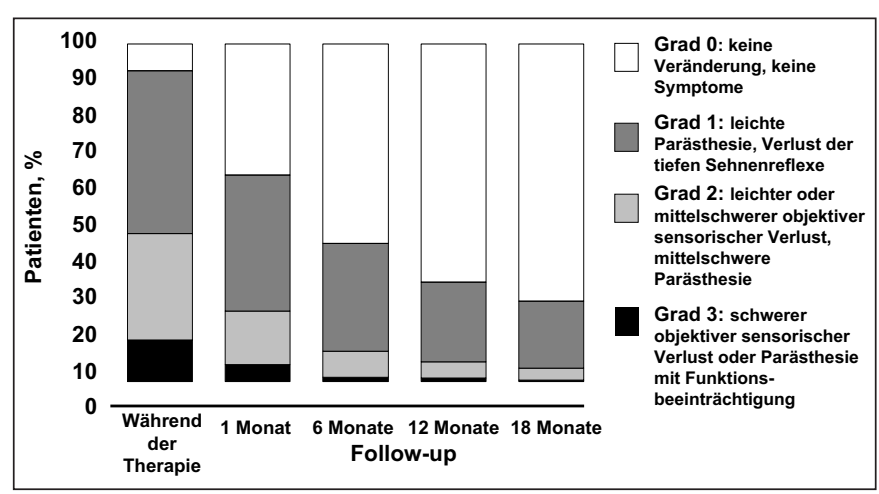

Abb. 2. MOSAIC: Periphere sensorische Neuropathie.

Grad 3/4 waren im FOLFOX4-Arm Neutropenie, Erbrechen und Diarrhoe. Eine Neutropenie vom Grad 3/4 war bei zusätzlicher Oxaliplatin-Gabe zwar häufiger als im Kontrollarm (41,1 vs. 4,7\%), die Rate daraus resultierender Komplikationen (Fieber, Infektionen) war jedoch mit beiden Regimen vergleichbar (1,8 vs. $0,2 \%)$. Häufigste Nebenwirkung unter FOLFOX4 war erwartungsgemäß die durch Oxaliplatin bedingte periphere Neuropathie $12,4 \%$ (Grad 3). Sie war jedoch in der Regel rasch reversibel (Abb. 2). Die therapiebedingte Mortalität in der MOSAIC-Studie war mit je $0,5 \%$ in beiden Gruppen niedrig.

\section{Vorteil von FOLFOX4 auch im Stadium II}

Ein weiteres wichtiges Ergebnis der MOSAIC-Studie ist die Tatsache, dass die Teilnehmer unabhängig vom Tumorstadium von der adjuvanten Therapie mit FOLFOX4 profitierten. Das belegt eine auf der diesjährigen ASCO-Tagung vorgestellte Subgruppenanalyse der 899 Patienten im Stadium II [16]: Während sich die Reduktion des Rezidivrisikos bei Patienten im Stadium III auf $24 \%$ belief, fiel sie
Tab. 1. MOSAIC-Studie: Verbesserung des krankheitsfreien Überlebens nach 3 Jahren um absolut 5\% bei Hochrisikopatienten im Stadium II [16]

\begin{tabular}{lll}
\hline Parameter & FOLFOX4 & FU/FS \\
\hline Patientenzahl & 286 & 290 \\
Zahl der Ereignisse & $40(14,0 \%)$ & $54(18,6 \%)$ \\
Wahrscheinlichkeit des ereignisfreien & & \\
$\quad$ Überlebens nach 3 Jahren & $84,9 \%$ & $79,8 \%$ \\
Hazard Ratio & & 0,72 \\
Relative Risikoreduktion & & $28 \%$ \\
& & \\
\hline
\end{tabular}

Tab. 2. MOSAIC-Studie: Schwere Sicherheitsereignisse waren bei Patienten im Stadium II seltener als bei Patienten im Stadium III [16]

\begin{tabular}{lllc}
\hline Stadium & & II & III \\
\hline Patientenzahl & & 891 & 1328 \\
Patienten mit SAE & FOLFOX4 & $18,8 \%$ & $25,4 \%$ \\
& 5-FU/FS & $13,5 \%$ & $15,3 \%$ \\
Therapieabbruch & & & \\
wegen Toxizität & FOLFOX4 & $12,1 \%$ & $16,0 \%$ \\
& 5-FU/FS & $5,8 \%$ & $5,3 \%$ \\
Tod unter Therapie & & & $0,8 \%$ \\
& FOLFOX4 & $0,2 \%$ & $0,5 \%$ \\
Schwere & 5-FU/FS & $0,7 \%$ & $33,7 \%$ \\
Sicherheitsereignisse & & & $17,6 \%$ \\
& FOLFOX4 & $24,2 \%$ & \\
\hline
\end{tabular}

$\mathrm{SAE}=$ Serious adverse events.

bei Patienten im Stadium II mit 20\% etwas geringer aus. Die Autoren weisen jedoch darauf hin, dass Kolonkarzinome im Stadium II eine heterogene Gruppe darstellen und daher neben dem Nodalstatus weitere Faktoren für die Risikostratifizierung herangezogen werden sollten. Identifizierte man Patienten im Stadium II mit mindestens einem weiteren Risikofaktor (z.B. T4, Obstruktion oder geringer Differenzierungsgrad), so konnte die Wahrscheinlichkeit für das krankheitsfreie Überleben nach 3 Jahren durch FOLFOX4 um absolut 5\% im Vergleich zum Kontrollarm gesteigert werden (Tab. 1). Das entspricht einer relativen Risikoreduktion durch die zusätzliche Oxaliplatin-Gabe um $28 \%$, die die für Patienten im Stadium III noch übertrifft. Auch im Stadium II sollte daher anhand des individuellen Rezidivrisikos durchaus eine adjuvante Therapie mit dem FOLFOX4-Regime in Erwägung gezogen werden, resümieren Hickish und Mitarbeiter [16]. Diese Empfehlung ist auch deswegen gerechtfertigt, weil schwere Beeinträchtigungen der Sicherheit im Studienverlauf (Inzidenz schwerwiegender Nebenwirkungen, Abbruch und/oder Tod) im Stadium II noch seltener registriert wurden als im Stadium III (19,8 vs. 25,6\%; Tab. 2 ). 
Das FOLFOX4-Regime ist damit auf dem besten Weg, sich als neuer Standard in der adjuvanten Chemotherapie des Kolonkarzinoms zu etablieren. Auch der Einwand, dass bislang keine langfristigen Gesamt-Überlebensdaten vorliegen, ist wenig stichhaltig, wie eine von D.J. Sargent, Mayo Clinic Rochester, vorgestellte Analyse großer Phase-III-Studien zur adjuvanten Therapie des Kolonkarzinoms belegt [23]. Zwar war die 5-Jahres-Überlebensrate bislang traditioneller Endpunkt in den großen adjuvanten Therapiestudien. Würde dieser Parameter jedoch zukünftig durch die Rate für das krankheitsfreie Überleben nach 3 Jahren ersetzt, so ließen sich therapeutische Fortschritte zum Wohle betroffener Patienten rascher in die klinische Praxis einführen. Voraussetzung ist allerdings, dass beide Parameter korrelieren, dass also die Rezidivfreiheit 3 Jahre nach der Operation prädiktiv für das weitere Überleben ist, erläuterte Sargent. Um diese Hypothese zu überprüfen, analysierte seine Arbeitsgruppe die individuellen Daten von mehr als 17000 Patienten mit Kolorektalkarzinomen aus 17 adjuvanten Therapiestudien. Sie waren in insgesamt 39 Armen mit auf 5-FU basierenden Regimen behandelt worden oder gehörten dem Kontrollarm ohne Therapie an.

\section{Verlässlicher Parameter: Krankheitsfreies Überleben}

Die ungeheure Informationsbasis macht deutlich, dass drei Viertel aller Rezidive beim Kolorektalkarzinom innerhalb der ersten 3 Jahre nach Tumorresektion auftreten. «Das Maximum der Rezidivrate ist bereits nach 1,5 Jahren erreicht. Danach sinkt die Wahrscheinlichkeit für eine Rezidivierung sehr rasch $a b »$, berichtete Sargent. Und wenn Rezidive verhindert werden, verbessert sich auch die Heilungschance: Entsprechend konnte eine sehr enge Korrelation zwischen der Rate für das krankheitsfreie Überleben nach 3 Jahren und der 5-Jahres-Gesamt-Überlebensrate nachgewiesen werden, sodass Sargent den ersten Parameter als «exzellenten Prädiktor» für das Gesamt-Überleben qualifizierte. In 22 vergleichenden Analysen von krankheitsfreiem und Gesamt-Überleben ergab sich in 19 Fällen für beide Parameter eine identische Aussage. Lediglich bei 3 Analysen zeigte sich ein signifikanter Vorteil nur für das krankheitsfreie Überleben. Gerade in diesen nicht übereinstimmenden Fällen war der Benefit der adjuvanten Therapie jedoch nur von marginaler Signifikanz. «Ein gering ausgeprägter Nutzen beim krankheitsfreien Überleben resultiert demnach nicht zwangsläufig in einem verbesserten Gesamt-Überleben», kommentierte Sargent. Insgesamt erlaubt das von seiner Arbeitsgruppe entwickelte Modell anhand der Therapieeffekte auf das krankheitsfreie Überleben eine gute Vorhersage des Gesamt-Überlebens. Seiner Meinung nach ist diese Variable daher als geeigneter Endpunkt anzusehen, der bevorzugt eingesetzt werden sollte, um die Erkenntnisse klinischer Studien rascher in die tägliche Praxis zu implementieren. Auf Basis dieser stringenten Analyse hat das Oncology
Drug Advisory Committee (ODAC), ein Beratungsgremium der amerikanischen Zulassungsbehörde FDA (Federal Drug Administration), kürzlich die einstimmige Empfehlung ausgesprochen, das krankheitsfreie Überleben als validen Parameter bei der Bewertung neuer Zulassungsanträge anzusehen.

\section{Irinotecan-Regime: Kein Benefit in der Adjuvanz}

Im Gegensatz zur zusätzlichen Gabe von Oxaliplatin zu 5-FU/FS haben Kombinationsregime mit Irinotecan bislang keinen Vorteil in der adjuvanten Therapie gezeigt. So fiel die von Leonard Saltz, Memorial Sloan-Kettering Cancer Center, vorgestellte Studie CALBG C89803 der Cancer and Leukemia Group B, in der das 5-FU/FS-Bolusregime nach Roswell Park über 4 Zyklen mit dem IFL- oder Saltz-Regime über 6 Zyklen verglichen wurde, in doppelter Hinsicht negativ aus [21]. In die Studie waren zwischen April 1999 und April 2001 insgesamt 1264 Patienten im Stadium III aufgenommen worden. Die Rekrutierung war dann aber wegen der erhöhten Toxizität im experimentellen IFL-Arm vorzeitig gestoppt worden: Therapiebedingte Todesfälle $(2,8$ vs. $1,0 \% ; p=0,008)$ und febrile Neutropenien ( 4 vs. $1 \% ; p=0,0005$ ) wurden mit dem Saltz-Regime signifikant häufiger registriert als im Kontrollarm. Auch hinsichtlich der Effektivität konnte die IrinotecanKombination nicht überzeugen: Nach 2,6-jährigem Follow-up zeigte sich beim ereignisfreien Überleben kein Vorteil im Vergleich zum Kontrollarm. «Die jetzt vorgestellten Ergebnisse sind endgültig, und C89803 kann daher nicht als positive Studie betrachtet werden», resümierte Saltz die Ergebnisse. Eric van Cutsem, University Hospital Gasthuisberg, Leuven, kam deshalb in seinem Diskussionsbeitrag zu dem eindeutigen Schluss: «Das IFL-Bolusregime sollte in der adjuvanten Therapie des Kolorektalkarzinoms nicht eingesetzt werden. Derzeit hat von den Kombinationsregimen nur FOLFOX4 seine Effektivität in der adjuvanten Situation belegt». Um die Rolle von Irinotecan zu klären, muss laut van Cutsem die Studie PETACC 3 (Pan European Trials in Adjuvant Colorectal Cancer) mit einem Irinotecan-haltigen 5-FU/FS-Infusionsregime abgewartet werden.

\section{Die Zukunft der adjuvanten Therapie}

Nach der Präsentation von X-ACT (Xeloda Adjuvant Colon Cancer Trial) werden 5-FU/FS-Regime zukünftig wohl ohnehin ihren Platz zugunsten der oralen Fluoropyrimidine räumen müssen. Für die Studie wurden 1987 Patienten mit einem Kolonkarzinom im Stadium III nach Resektion zum 5-FU/FSBolusregime der Mayo-Klinik oder zu Capecitabin randomisiert [5]. Nach median 3-jähriger Beobachtungszeit zeigte sich hinsichtlich des krankheitsfreien Überlebens als primärem Endpunkt zumindest eine Äquieffektivität beider Regime, tendenziell sogar ein Vorteil zugunsten des oralen Fluoropyri- 
midins gegenüber dem Kontrollarm (64,2 vs. 60,6\%), berichtete J. Cassidy, Universität Glasgow. Darüber hinaus überzeugte die Substanz aufgrund der besseren Verträglichkeit. Aufgrund dieser Ergebnisse sollte Capecitabine zukünftig 5-FU/FS in der adjuvanten Therapie des Kolonkarzinoms ersetzen, resümierte van Cutsem. Er sieht die Zukunft der adjuvanten Therapie in der Kombination oraler Fluoropyrimidine mit Zytostatika wie Oxaliplatin und mit neuen «targeted therapies» wie Bevacizumab, Cetuximab und Tyrosinkinase(TK)-Inhibitoren des EGFR (epidermal growth factor receptor).

\section{Zahlreiche neue Kombinationsstudien mit molekularen Therapien}

Derartige Studien sind bereits angelaufen: So wird in der Studie 5202 der Eastern Cooperative Oncology Group (ECOG) bei Hochrisikopatienten im Stadium II das FOLFOX-Regime mit der Kombination aus FOLFOX und dem antiangiogenetisch wirkenden Bevacizumab verglichen. Gleichzeitig findet in dieser Studie mithilfe molekularer Prädiktoren (z.B. Mikrosatelliteninstabilität) eine Risikostratifizierung statt, wobei Patienten niedrigen Risikos einem Kontrollarm mit alleiniger Beobachtung zugeteilt werden. Für die Studie C-08 des National Surgical Adjuvant Bowel and Breast Project (NSABP) sollen insgesamt 2300 Patienten im Stadium II und III zu einem Kontrollarm mit einem modifizierten FOLFOX7-Regime (mFOLFOX7) oder zu einem experimentellen Arm mit mFOLFOX7 plus Bevacizumab randomisiert werden. Die dreiarmige Studie BO17920 schließlich vergleicht FOLFOX4 mit FOLFOX4 plus Bevacizumab und mit der Kombination Capecitabin plus Oxaliplatin (XELOX). Weitere Studien evaluieren die Effektivität von Cetuximab in der adjuvanten Therapie, ebenfalls in Kombination mit FOLFOX4 oder mit Capecitabin/Oxaliplatin.

\section{Oxaliplatin-Regime: Standard in der First-line-Therapie}

Die hohe Toxizität des Saltz-Regimes war bereits 2001 in der Intergroup-Studie N9741 des National Cancer Institutes (NCI) bei Patienten mit inoperablem, histologisch gesichertem fortgeschrittenem Kolorektalkarzinom aufgefallen [22]. In der ursprünglich sechsarmigen, mehrfach modifzierten Studie hatten sich schließlich nur 2 Vergleichsarme mit FOLFOX4- und Saltz-Regime behaupten können. Allerdings hatte auch letzteres wegen inakzeptabler Toxizität und hoher 60-Tage-Mortalitätsrate $(4,5 \%)$ durch Reduktion der Irinotecan-Dosis von 125 $\mathrm{mg} / \mathrm{m}^{2}$ auf $100 \mathrm{mg} / \mathrm{m}^{2}$ und der 5-FU-Dosis von $500 \mathrm{mg} / \mathrm{m}^{2}$ auf $400 \mathrm{mg} / \mathrm{m}^{2}$ (R-IFL) geändert werden müssen. Auf der ASCOTagung 2003 waren die Studienergebnisse nach 20-monatigem Follow-up vorgestellt worden, die die signifikante Überlegenheit des FOLFOX4-Arms gegenüber dem IFL-Regime bei fast allen Studienendpunkten deutlich gemacht hatten [12]: Sowohl
Tab. 3. Intergroup-Studie N9741: Über $70 \%$ der Patienten beider Studienarme erhielten eine Second-line-Therapie, davon über die Hälfte mit der jeweils anderen Substanz [13]

\begin{tabular}{lll}
\hline & $\begin{array}{l}\text { R-IFL } \\
(\mathrm{n}=151)\end{array}$ & $\begin{array}{l}\text { FOLFOX } \\
(\mathrm{n}=154)\end{array}$ \\
\hline Patienten mit 2nd-line-Therapie, \% & 72 & 71 \\
Irinotecan, \% & 21 & 53 \\
Oxaliplatin, \% & 54 & 18 \\
5-FU, \% & 34 & 21 \\
\hline
\end{tabular}

hinsichtlich Gesamt-Überlebenszeit (19,5 vs. 14,8 Monate) als auch hinsichtlich Ansprechraten (45 vs. 31\%) und Zeit bis zur Progression $(8,7$ vs. 6,9$)$ schnitt das Oxaliplatin-Regime bei gleichzeitig besserer Verträglichkeit günstiger ab und avancierte damit zum neuen Standard in der First-line-Therapie des metastasierten Kolorektalkarzinoms.

Auf der diesjährigen ASCO-Tagung wurden die Endresultate von 355 Patienten der NCI-Studie vorgestellt, die zu FOLFOX4 oder dem modifizierten R-IFL-Regime randomisiert wurden. Sie sprechen ebenfalls für die Oxaliplatin-Kombination [13]. So konnte die mediane Überlebenszeit nach 22,5monatigem Follow-up mit dem FOLFOX4-Regime bis auf 18,8 Monate verlängert werden - gegenüber nur 16,3 Monaten mit R-IFL. Dieser Unterschied ist umso bemerkenswerter, kommentieren Goldberg et al., als dass jetzt in der Secondline-Therapie die Mehrzahl aller Patienten das zuvor in der First-line-Therapie nicht eingesetzte Zytostatikum (d.h. auch Oxaliplatin im R-IFL-Arm) erhielt (Tab. 3), während der Zugang zu Oxaliplatin in früheren Studienphasen noch limitiert und eine effektive Second-line-Therapie daher nur eingeschränkt möglich war. Auch bei Zeit bis zur Progression (10,0 vs. 6,9 Monate; $\mathrm{p}<0,0001$ ) und Responseraten (47 vs. $32 \% ; p=0,0006)$ ergaben sich signifikante Vorteile zugunsten von FOLFOX4.

Die Toxizität war in beiden Studienarmen nur mäßig ausgeprägt; allerdings waren Neutropenie und Parästhesien mit dem FOLOFOX4-Regime signifikant häufiger. Auch wenn die Verträglichkeit der Irinotecan-haltigen Kombiation nach Dosisreduktion deutlich verbessert werden konnte, ist das RIFL-Regime wegen seiner gegenüber FOLFOX4 geringeren Effektivität für die First-line-Therapie des fortgeschrittenen Kolorektalkarzinoms nicht als optimal zu betrachten, resümieren die Autoren.

\section{OPTIMOX-Studien: Oxaliplatin-Regime weiter verbessern}

Das FOLFOX4-Regime hat sich als hoch effektive und insgesamt verträgliche Kombination bewährt. Allerdings kann die mit Oxaliplatin mögliche kumulative sensorische Neuropathie einen Behandlungsabbruch bei respondierenden Patien- 
526 Patienten mit unbehandeltem MCRC

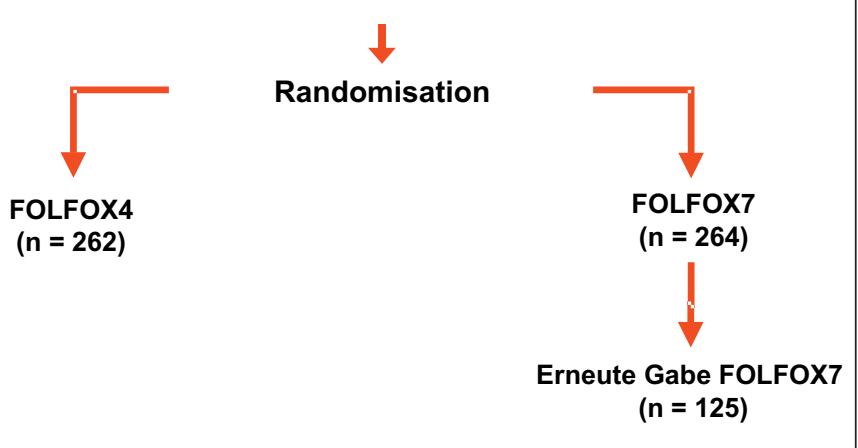

Abb. 3. Design der OPTIMOX1-Studie [6].

ten erforderlich machen. Da die Lebenserwartung von Patienten mit fortgeschrittenem Kolorektalkarzinom mittlerweile deutlich verlängert wurde und Patienten daher sehr viel länger behandelt werden, ist diese kumulative Nebenwirkung laut Paulo M. Hoff, M.D. Anderson Cancer Center, durchaus als relevant anzusehen. Limitierend kann weiterhin die durch den 14-tägig applizierten 5-FU-Bolus induzierte Neutropenie sein.

OPTIMOX1, eine Phase-III-Studie an 526 Patienten mit inoperablem oder metastasiertem Kolorektalkarzinom, hatte daher das Ziel, die Effektivität des bewährten Regimes weiter zu steigern und gleichzeitig Nebenwirkungen zu minimieren. In der zweiarmigen Studie wurde das bekannte FOLFOX4Regime (Arm A) mit dem FOLFOX7-Schema verglichen, bei dem eine höhere Oxaliplatin-Dosis $\left(130 \mathrm{mg} / \mathrm{m}^{2}\right)$ mit einem 5-FU/FA-Regime ohne 5-FU-Bolus kombiniert wird. Nach 6 Gaben FOLFOX7 wird dann mit einem vereinfachten 5-FU/FS-Regime (s5LV5FU2) als Erhaltungstherapie weiterbehandelt, nach 12 Zyklen - bei Progression bereits früher wieder auf FOLFOX7 umgestellt (Arm B). Laut den von de Gramont und Mitarbeitern vorgestellten Ergebnissen sind beide Regime ähnlich effektiv [6]: Die Gesamt-Überlebenszeiten sind mit 20 Monaten für FOLFOX4 und 21,6 Monaten für FOLFOX7 vergleichbar; progressionsfreies Überleben (9,2 vs. 9,0 Monate), Dauer der Tumorkontrolle (9,2 vs. 9,7 Monate) und Gesamtansprechraten (58,5 vs. 58,3\%) nahezu identisch. Das Gesamtüberleben war in den Zentren, die die höchsten Anteile an Reinduktionen im FOLFOX7-Arm hatten, am längsten. Das angestrebte Ziel, die Therapieergebnisse durch Erhöhung der Oxaliplatin-Dosis weiter zu verbessern, wurde daher leider nicht erreicht, bedauerte Hoff in seiner Diskussion der Studienergebnisse. Allerdings hat die Studie klar gezeigt, dass eine Oxaliplatin-Therapiepause bei Erhalt der Wirksamkeit und verbessertem Toxizitätsprofil möglich ist, betonte er. So überzeugt das FOLFOX7-Regime durch eine bessere Verträglichkeit mit geringeren Neutropenie- und Neuropathieraten, und es wird von den Autoren als patientenfreundlicher beschrieben.

\section{FOLFOX: Günstig auch für gefährdete Patienten}

Auch für ältere Patienten ( $>75$ Jahre), die oft aus klinischen Studien ausgeschlossen werden, sind die Oxaliplatin-haltigen Kombinationen geeignet, wie eine exploratorische Analyse im Rahmen von OPTIMOX 1 belegt. Von den 37 über 75-jährigen Patienten in OPTIMOX1 erhielten 20 das FOLFOX4Standardregime, 17 FOLFOX7 [7]. Die Gesamtansprechraten waren mit $65 \%$ für FOLFOX4 und 53\% für FOLFOX7 vergleichbar. Ähnliches gilt für das progressionsfreie Überleben mit 7,6 bzw. 9,4 Monaten ( $\mathrm{p}=0,96)$ und das Gesamt-Überleben mit 14,9 bzw. 25,1 Monaten ( $\mathrm{p}=0,15)$. Die Verträglichkeit des FOLFOX4-Regimes bei über 75-jährigen Patienten ist ähnlich günstig wie bei den jüngeren Studienteilnehmern; lediglich Neutropenien treten deutlich häufiger auf. FOLFOX4 und FOLFOX7 unterscheiden sich hinsichtlich ihres Toxizitätsprofils bei den über 75-Jährigen kaum, wobei die Rate an Neutropenien vom Grad 3/4 mit FOLFOX7 niedriger ist. Insgesamt bleibt das günstige Nutzen-Risiko-Profil der Oxaliplatin-haltigen Regime damit auch bei älteren Patienten erhalten, sodass die Autoren diese in ausgewählten Kollektiven mit gutem Performance-Status als sicher erachten.

Ebenfalls effektiv sind FOLFOX-Regime bei Patienten mit ungünstiger Prognose, definiert anhand eines stark erhöhten Spiegels an alkalischer Phosphatase - so eine weitere exploratorische Analyse von OPTIMOX1 mit insgesamt 67 Teilnehmern [26]. Die Gesamtresponseraten waren mit 48,5\% im FOLFOX4-Arm und 63,4\% im FOLFOX7-Arm hoch und unterschieden sich nicht von denen im Gesamtkollektiv. Gesamt-Überlebenszeit und progressionsfreie Zeit waren mit 14 versus 11 Monaten bzw. 6,7 versus 6,5 Monaten in beiden Armen vergleichbar. Demnach kann Oxaliplatin das Überleben im Vergleich zu einer reinen 5-FU/FS-Therapie auch in diesem Kollektiv verlängern. Es ist jedoch mit rund 12 Monaten deutlich kürzer als der sonst mögliche Medianwert von rund 21 Monaten.

\section{Mehr Lebensqualität durch therapiefreies Intervall?}

In OPTIMOX2 soll der Stellenwert der zwischen den FOLFOX7-Zyklen verabreichten LV5FU2-Erhaltungstherapie geklärt werden. Zu diesem Zweck erfolgt eine Randomisierung zu dem gleichen Regime wie in Arm B von OPTIMOX 1 oder zu einem «Stop-and-go»-Verfahren mit 6 Zyklen FOLFOX7, anschließender Therapiepause und erneuter Gabe von 6 Zyklen FOLFOX7 (Abb. 3). Davon verspricht man sich eine mit bessere Lebensqualität und eine Minimierung von Resistenzrisiko und Kosten. Im Rahmen einer Phase-II-Studie untersuchten T. André, Hôpital Tenon, Paris, und Mitarbeiter gleichzeitig als neue Strategie den Einsatz des antineoplastisch wirkenden, selektiven Cyclooxygenase-2-Hemmers Celecoxib im chemotherapiefreien Intervall des experimentellen Arms [2]. Bei bislang 42 evaluierbaren 
Patienten überzeugte dieses Konzept durch ein gutes Sicherheitsprofil. Die Responserate liegt mit 47\% - davon 2 Komplettremissionen (CR) - im üblichen Bereich. Das progressionsfreie Überleben in dieser Phase-II-Studie ist mit 6 Monaten jedoch enttäuschend kurz, kommentieren die Autoren. Zum Vergleich: In OPTIMOX1 betrug dieses Intervall im FOLFOX7-Arm 9 Monate. Ob die intermittierende Therapie mit chemotherapiefreien Intervallen eine Zukunft hat, muss die Phase III der OPTIMOX2-Studie zeigen. Grundsätzlich bietet es sich nach Ansicht der Autoren an, die Therapiepausen zur Applikation neuer molekularer Substanzen zu nutzen.

Dagegen bezeichnete Hoff ein chronomoduliertes Regime mit Oxaliplatin und 5-FU/FS (FFL4-10), das in der Studie 05963 der European Organization for Research and Treatment of Cancer zur Verbesserung von Effektivität und Verträglichkeit erprobt wurde, als für den Routineeinsatz wenig geeignet [10]. Das chronomodulierte Regime, das höhere Dosen bei weniger Toxizität ermöglichen soll, war bei 554 Patienten mit metastasiertem Kolorektalkarzinom mit dem FOLFOX2-Regime verglichen worden. Die Autoren hatten eine 10\%-ige Steigerung der 2-Jahres-Überlebensrate als primären Endpunkt durch die Chronomodulation erhofft. Mit Gesamt-Überlebenszeiten von 19,6 Monaten für FOLFOX2 und 18,7 Monaten für das chronomodulierte Regime hatten sich jedoch beide Therapien als vergleichbar effektiv herausgestellt. Progressionsfreies Überleben und Ansprechraten waren mit 8,4 Monaten bzw. gut $40 \%$ in beiden Armen praktisch identisch. Einziger Vorteil der Chronomodulation war die deutliche Reduktion der Neutropenierate von 25,3\% mit FOLFOX2 auf 7,3\%; ansonsten war die Toxizität in beiden Armen akzeptabel. Für das FOLFOX-Regime spricht jedoch die sehr viel einfachere Applikation, bei der die Infusionszyklen nicht an den zirkadianen Rhythmus angepasst werden müssen.

\section{Orale Prodrugs ersetzen 5-FU}

In der adjuvanten Therapie des Kolorektalkarzinoms ist die Prüfung oraler 5-FU-Prodrugs in Kombination mit Oxaliplatin gerade angelaufen. Anders beim metastasierten Tumor, für den bereits vielversprechende Ergebnisse zu derartigen Kombinationen vorliegen. So zeigt eine deutsche randomisierte Phase-II-Studie, dass Kombinationen von Capecitabin $\left(2 \times 1000 \mathrm{mg} / \mathrm{m}^{2} / \mathrm{Tag}\right.$, Tage $\left.1-14\right)$ mit Oxaliplatin $\left(70 \mathrm{mg} / \mathrm{m}^{2}\right.$ i.v. Tage 1 und 8; CAPOX) oder mit Irinotecan $\left(100 \mathrm{mg} / \mathrm{m}^{2}\right.$ i.v. Tage 1 und 8; CAPIRI) machbar sind und sich durch gute Verträglichkeit und hohe Aktivität auszeichnen. Wie auf der letztjährigen ASCO-Tagung berichtet, werden in der First-lineTherapie Gesamtresponseraten von 51,3\%, davon 6,6\% CR mit CAPOX und von 42,6\% (CR: 2,9\%) mit CAPIRI erreicht [14]. Auch in der Second-line-Therapie nach dem protokollarisch vorgesehenen Cross-over bei Progress waren beide Regime weiterhin effektiv und gut verträglich [15]. Die Re-
Tab. 4. Phase-III-Studie der AIO: Nebenwirkungsraten unter FUFOXversus CAPOX-Regime [3]

\begin{tabular}{|c|c|c|c|c|}
\hline & \multicolumn{4}{|c|}{ Patienten, \% } \\
\hline & \multicolumn{2}{|c|}{ Capox $(n=164)$} & \multicolumn{2}{|c|}{ FUFOX $(\mathrm{n}=145)$} \\
\hline & Grad 1/2 & Grad 3/4 & Grad 1/2 & Grad 3/4 \\
\hline Durchfall & 33 & 12 & 35 & 14 \\
\hline Übelkeit & 49 & 7 & 62 & 9 \\
\hline Erbrechen & 27 & 5 & 31 & 7 \\
\hline Neuropathie & 52 & 16 & 45 & 25 \\
\hline Stomatitis & 12 & 1 & 16 & 3 \\
\hline HFS & 29 & 1 & 27 & 0 \\
\hline Schmerz & 30 & 5 & 33 & 4 \\
\hline Fieber & 17 & 0 & 18 & 1 \\
\hline Infektion & 12 & 3 & 14 & 4 \\
\hline
\end{tabular}

sponserate betrug mit CAPIRI 21\%, mit CAPOX 13\%; bei jeweils etwa der Hälfte der Patienten wurde eine Stabilisierung erreicht. Schwere Nebenwirkungen (Grad 3/4) waren insgesamt selten.

Diese Studie war die Basis für eine deutsche Phase-III-Studie der Arbeitsgemeinschaft internistischer Onkologen (AIO), in der die CAPOX-Kombination mit dem 5-FU/FS-Infusionsregime der AIO plus Oxaliplatin (FUFOX) bei 399 Patienten mit metastasiertem Kolorektalkarzinom als First-line-Therapie verglichen wird. Der auf der ASCO-Tagung 2004 vorgestellten Sicherheitsanalyse zufolge ist das Toxizitätsprofil der Zweierkombination mindestens ebenso günstig wie das des gut verträglichen FUFOX-Regimes [Arkenau et al.]. Häufigkeit und Schweregrad gastrointestinaler Nebenwirkungen und des Hand-Fuß-Syndroms sind in beiden Gruppen vergleichbar. Eine schwere sensorische Neuropathie vom Grad 3/4 tritt unter CAPOX sogar deutlich seltener auf als mit dem FUFOX-Regime (Tab. 4). Eine Hämatotoxizität vom Grad 3/4 war insgesamt selten; häufiger wurden lediglich Neutropenien im FUFOX-Arm dokumentiert.

Drei verschiedene Oxaliplatin-Kombinationen werden in der Studie TREE 1 bei 150 Patienten mit metastasiertem Kolorektalkarzinom untersucht. Hier wird das klassische FOLFOX4-Regime mit CapeOx (130 mg/m² Oxaliplatin i.v. Tag 1 plus $1000 \mathrm{mg} / \mathrm{m}^{2}$ Capecitabin 2x/Tag, 14 Tage alle 3 Wochen) und dem bFOL-Regime mit Oxaliplatin $\left(85 \mathrm{mg} / \mathrm{m}^{2}\right.$, Tag 1, 15) plus 5-FU-Bolusregime (500 mg/m² Tag 1, 8, 15 alle 4 Wochen) verglichen [27]. Die Gesamtansprechraten liegen zwischen $38 \%$ (bFOL) und 52\% (FOLFOX) ohne signifikante Unterschiede zwischen den drei Armen. Hinsichtlich Verträglichkeit erwies sich das bFOL-Regime als am günstigsten; im CapeOx-Arm mussten 38\% der Patienten die Therapie toxizitätsbedingt in den ersten 12 Wochen unterbrechen.

Entsprechend wurde die Capecitabin-Dosis für die Studie TREE 2 auf $850 \mathrm{mg}$ zweimal täglich abgesenkt. Diese Studie, für die 223 Patienten rekrutiert wurden, gehört bereits einer neuen Generation an, da die 3 Therapieregime aus TREE 1 
Tab. 5. Hohe Responseraten in der Phase-II-Studie mit der Kombination aus FOLFOX4 plus Cetuximab [24]. Bei 7 Patienten erfolgte eine Resektion von Lebermetastasen.

\begin{tabular}{lcc}
\hline Gesamtansprechraten & N / \% & $95 \%$ CI \\
\hline Komplettes Ansprechen & $2 / 5 \%$ & $1-16$ \\
Partielles Ansprechen & $32 / 76 \%$ & $61-88$ \\
Stabilisierung & $7 / 17 \%$ & $7-32$ \\
Progression & $1 / 2 \%$ & $1-13$ \\
\hline
\end{tabular}

mit Bevacizumab kombiniert werden. Die jetzt präsentierten Sicherheitsdaten aus TREE 2 sind sehr beruhigend und sprechen für das akzeptable Toxizitätsprofil aller drei Arme - einschließlich CapeOx. Mit Spannung werden jetzt die Effektivitätsdaten dieser Studie erwartet, da eine weitere Überlebensverlängerung auf bis zu 24 Monate angenommen wird. Damit hätte man die Lebenserwartung von Patienten mit fortgeschrittenem Kolorektalkarzinom in den vergangenen Jahren etwa vervierfacht, betrug doch das mittlere Überleben noch in den achtziger Jahren bei alleiniger Supportivtherapie nur rund 6 Monate.

\section{Vielversprechende Ergebnisse mit molekularen Therapieansätzen}

Welche beeindruckenden Ergebnisse mit Kombinationen der klassischen Chemotherapie plus Biologicals zu erreichen sind, zeigt eine internationale Phase-II-Studie, in der das FOLFOX4-Regime zusammen mit dem monoklonalen, gegen EGFR gerichteten Antikörper Cetuximab als Firstline-Therapie verabreicht wurde [24]. In die Studie wurden bislang 43 Patienten mit fortgeschrittenem Kolorektalkarzinom und Nachweis einer EGFR-Expression in Tumorgewebe oder Metastasen aufgenommen. Sie erhielten als First-line-Therapie zusätzlich zum FOLFOX4-Regime Cetuximab in einer Dosis von $400 \mathrm{mg} / \mathrm{m}^{2}$ i.v. an Tag 1; anschließend wurde Cetuximab wöchentlich in einer Dosis von $250 \mathrm{mg} / \mathrm{m}^{2}$ verabreicht.

Die Effektivität der innovativen Kombination bei den 42 evaluierbaren Patienten ist ausgesprochen hoch: Insgesamt sprachen 34 Patienten $(81 \%)$ an, zwei davon komplett. Werden auch Stabilisierungen berücksichtigt, so steigt die Tumorkontrollrate auf 98\% an (Tab. 5). Das Sicherheitsprofil der Kombination beschreiben die Autoren als akzeptabel: Als häufigste Nebenwirkungen vom Grad 3/4 wurden Diarrhöen (26\%), akneformer Ausschlag (21\%) und Neutropenien (14\%) registriert. In seiner Diskussion der Studienergebnisse bezeichnete Saltz die erreichten Responseraten als sehr ermutigend und als gute Basis für eine Phase-III-Studie. Der routinemäßige Einsatz der FOLFOX4-Cetuximab-Kombination außerhalb klinischer Studien sei jedoch bislang noch nicht gerechtfertigt. Hier seien die Ergebnisse einer CALBG-Studie an $1100 \mathrm{~Pa}-$ tienten abzuwarten, in der die Kombination von mFOLFOX6 mit oder ohne Cetuximab mit dem FOLFIRI-Regime, ebenfalls mit oder ohne Antikörper, in der First-line-Therapie des fortgeschrittenen Kolorektalkarzinoms verglichen werde.

\section{Geprüft werden Antikörper und «small molecules»}

Ebenfalls durch sehr hohe Ansprechraten fällt eine weitere Phase-II-Studie auf, in der das FOLFOX4-Regime mit dem EGFR-TK-Inhibitor Gefitinib (IFOX) kombiniert wurde [8]. Hier erfolgte eine Stratifizierung der insgesamt 63 Patienten: 31 Teilnehmer waren nicht vortherapiert (Gruppe A), weitere 27 hatten bereits eine Irinotecan-haltige First-line-Therapie erhalten (Gruppe B). In Gruppe A sprachen 23 Patienten (77\%) partiell auf die Therapie an, bei weiteren $20 \%$ gelang eine Tumorstabilisierung. In Gruppe B lag die Responserate bei $29 \%$, die Stabilisierungsrate bei 58\%. Die Zeit bis zur Progression beträgt 9,5 bzw. 5,2 Monate. Das IFOX-Regine ist allerdings durch eine höhere Rate gastrointestinaler Nebenwirkungen (Diarrhöe, Übelkeit, Erbrechen) im Vergleich zu FOLFOX4 charakterisiert. Die hohe Diarrhörate von 54\% sollte laut Saltz auf jeden Fall zur Vorsicht bei der weiteren Erprobung von IFOX mahnen. Bislang ist noch unklar, ob die bei Patienten mit Bronchialkarzinom nachgewiesenen aktivierenden Mutationen im EGFR, die zu einer sehr starken Tumorschrumpfung und lang anhaltenden Remissionen führen, auch beim Kolorektalkarzinom auftreten. Die Hypothese, dass eine EGFR-Expression im Tumor Voraussetzung für das Ansprechen auf EGFR-Antagonisten ist, kann heute laut Saltz als widerlegt gelten. Er bezeichnete es als falsch und zynisch, Patienten eine gegen EGFR gerichtete Therapie nur aufgrund einer fehlenden EGFR-Expression vorzuenthalten.

\section{Oxaliplatin ermöglicht Resektion von Lebermetastasen}

Bei mehr als der Hälfte aller Patienten mit kolorekatalem Karzinom treten im Erkrankungsverlauf Lebermetastasen auf. Lediglich die chirurgische Resektion, die allerdings primär nur in maximal einem Viertel der Fälle möglich ist, bietet den Betroffenen eine Heilungschance. Als effektiv hat sich jedoch die neoadjuvante Chemotherapie erwiesen, die zu Downstaging und verbesserter Resektabilität führt. So gelang in der Sequenzstudie von Tournigand et al. mit dem FOLFOX-Regime als First-line-Therapie bei 21 der 59 Responder eine chirurgische Entfernung zuvor inoperabler Lebermetastasen; 13 von ihnen konnten R0-reseziert werden [25]. Nach einer First-line-Therapie mit dem FOLFIRI-Regime war dagegen nur bei 8 von 61 Respondern eine Resektion möglich. Laut einer retrospektiven Analyse entspricht das Überleben R0-resezierter Patienten mit rund 70 Monaten dem von Patienten, deren Lebermetastasen primär chirurgisch entfernt werden konnten [9]. 
Problematisch ist allerdings das Vorgehen bei metastasierten Patienten nach Progression unter einer First-line-Therapie, da die Chancen für ein Ansprechen auf Oxaliplatin in der Second line geringer sind. In solchen Fällen könnte eine lokoregionäre Infusionstherapie über die Arteria hepatica (HAI) erfolgreicher sein, da das Tumorgewebe so einer höheren Zytostatikakonzentration ausgesetzt wird [17]. Die Autoren erprobten dieses Vorgehen bei 44 Patienten mit alleinigen, aber inoperablen Lebermetastasen, die auf eine First-line-Chemotherapie, meist mit Irinotecan-Kombinationen, nicht mehr angesprochen hatten. Die HAI wurde mit einer systemischen Chemotherapie kombiniert, um so eine effektive Kontrolle von Lebermetastasen und extrahepatischer Erkrankung zu erreichen.

21 Patienten erhielten neben der HAI mit Floxuridin und Dexamethason eine systemische Chemotherapie mit Oxaliplatin und 5-FU/FS, 23 weitere Patienten zusätzlich zur HAI die Kombination aus Oxaliplatin/Irinotecan. Im Gesamtkollektiv war die Ansprechrate mit $82 \%$ hoch, obwohl fast drei Viertel der Teilnehmer unter der vorherigen Therapie progredient geworden waren. In $7 \%$ der Fälle gelang eine pathologische Komplettremission. Bei 9 Patienten (20\%) konnten die Lebermetastasen bereits erfolgreich reseziert werden; bei weiteren 7 Patienten ist eine Operation möglich. Nach einem medianen Follow-up von jetzt 21 Monaten beträgt die progressionsfreie Zeit bei den systemisch mit Oxaliplatin/5-FU behandelten Patienten 19 Monate, in der Oxaliplatin/IrinotecanGruppe 15 Monate. Die Gesamt-Überlebenszeiten erstrecken sich über mittlerweile 23 bzw. 36 Monate und sind damit als exzellent für dieses Patientenkollektiv zu werten. Die Autoren empfehlen jetzt eine randomisierte Vergleichsstudie, in der ihr Ansatz mit einer alleinigen neoadjuvanten systemischen Chemotherapie verglichen wird.

\section{Pankreaskarzinom: Gem0x verbessert Therapieergebnisse}

Zur Sitzung «Gastrointestinal (Noncolorectal) Cancer» unter Leitung von Emily K. Bergsland, University of California, San Francisco Cancer Center, und Charles D. Blanke, Oregon Health and Science University, und zur Postersitzung «Gastrointestinal (Noncolorectal) Cancer»

Seit Einführung von Gemcitabin - derzeit immer noch Standard in der Behandlung des Pankreaskarzinoms - versucht man eine Steigerung der unbefriedigenden Ansprech- und Überlebensraten durch Kombination mit weiteren Zytostatika $\mathrm{zu}$ erreichen. Am erfolgversprechendsten stellte sich in einer Phase-II-Studie die als GEMOX bezeichnete Kombination mit Gemcitabin (1000 mg/m² i.v. als 100-min-Infusion, Tag 1) und Oxaliplatin (100 mg/m² i.v., Tag 2) alle 2 Wochen heraus [18]: Bei guter Verträglichkeit wurden eine hohe Ansprechrate von $30 \%$, eine klinische Benefitrate von fast $40 \%$, ein medianes progressionsfreies Intervall von 5,3 und eine Gesamt-
Tab. 6. Therapieergebnisse der Phase-III-Studie von GERCOR/GISCAID mit der Gemcitabin-Monotherapie versus GEMOX-Kombination [19]

\begin{tabular}{llll}
\hline & GO & G & P-Wert \\
\hline Patienten & 157 & 156 & \\
Ansprechrate* & $26,8 \%$ & $17,3 \%$ & 0,04 \\
Überleben & 9,0 Monate & 7,1 Monate & 0,13 \\
Progressionsfreies Überleben & 5,8 Monate & 3,7 Monate & 0,038 \\
1-Jahres-Überlebensrate & $34,7 \%$ & $27,8 \%$ & \\
8-Monats-Überlebensrate & $56,5 \%$ & $45,3 \%$ & 0,048 \\
\hline
\end{tabular}

*Remissionen wurden nicht zentral begutachtet, schließen geschätzte partielle Remissionen ein.

Überlebenszeit von immerhin 9,2 Monaten erreicht. Die 1-Jahres-Überlebensraten von $47 \%$ bei Patienten mit lokal fortgeschrittenem und von $26 \%$ beim metastasierten Pankreaskarzinom lagen in dieser Studie deutlich über denen der Gemcitabin-Monotherapie.

Diese ermutigenden Daten rechtfertigten die Initiierung einer Phase-III-Studie, in der die neue Kombination mit Gemcitabin als Monotherapie verglichen wurde. Für die von der französischen GERCOR- und der italienischen GISCAD-Gruppe durchgeführte Studie wurden zwischen März 2001 und Februar 2003313 Patienten mit histologisch gesichertem Pankreasadenokarzinom rekrutiert [19]. 156 Patienten wurden randomisiert dem Kontrollarm mit Gemcitabin $\left(1 \mathrm{~g} / \mathrm{m}^{2}\right.$ wöchentlich als 30-minütige Infusion), 157 Patienten dem experimentellen Arm mit der GEMOX-Kombination zugeteilt. Rund 30\% der Patienten in beiden Armen wiesen einen lokal fortgeschrittenen, 70\% einen metastasierten Tumor auf. Primäres Ziel der Studie war eine 20\%-ige Zunahme der Überlebensrate von 30 auf $50 \%$ nach 8 Monaten. Sekundäre Wirkparameter waren progressionsfreies Überleben, Ansprechrate, klinischer Benefit, Verträglichkeit und Lebensqualität.

\section{Überlebensverlängerung um 2 Monate}

Die bereits in der Phase-II-Studie beobachtete eindrucksvolle Aktivität der GEMOX-Kombination bestätigte sich auch in dieser randomisierten Vergleichsstudie anhand der signifikant höheren Responserate im Vergleich zur Gemcitabin-Monotherapie (26,8 vs. 17,3\%; $p=0,04$; Tab. 6). Auch bei der klinischen Benefit-Rate zeigte sich ein signifikanter Vorteil zugunsten des Kombinationsregimes (38,2 vs. $26,9 \%$; $p=0,03$ ), wobei Patienten mit lokal fortgeschrittenem Tumor besonders stark profitierten (45,1 vs. 34,0\%). Darüber hinaus gelang eine erhebliche Verlängerung des progressionsfreien Überlebens von nur 3,7 Monaten unter Gemcitabin auf 5,8 Monate mit der Oxaliplatin-Kombination ( $\mathrm{p}=0,038)$.

Auch die Gesamt-Überlebenszeit konnte durch die Kombinationstherapie um rund 2 Monate verlängert werden (9,0 vs. 


\begin{tabular}{|c|c|c|}
\hline & $\mathbf{R}$ & $\begin{array}{l}\text { Arm A: } \\
\text { Gemcitabin } 1000 \mathrm{mg} / \mathrm{m}^{2} / 30 \mathrm{~min} \\
\text { qw x } 3 \text { alle } 4 \text { Wochen }\end{array}$ \\
\hline \multirow[t]{3}{*}{$\begin{array}{c}791 \\
\text { Patienten }\end{array}$} & $\begin{array}{l}\mathrm{O} \\
\mathrm{M} \\
\mathrm{I}\end{array}$ & $\begin{array}{l}\text { Arm B: } \\
\text { Gemcitabin } 1500 \mathrm{mg} / \mathrm{m}^{2} / 150 \mathrm{~min} \\
\text { qw } \times 3 \text { alle } 4 \text { Wochen }\end{array}$ \\
\hline & $\begin{array}{l}\mathbf{S} \\
\mathbf{1}\end{array}$ & \\
\hline & $\begin{array}{l}E \\
R \\
T\end{array}$ & $\begin{array}{l}\text { Arm C: } \\
\text { Gemcitabin } 1000 \mathrm{mg} / \mathrm{m}^{2} / 100 \mathrm{~min} \\
\text { Oxaliplatin } 100 \mathrm{mg} / \mathrm{m}^{2} / 120 \mathrm{~min} \\
\text { q } 14 \text { Tage }\end{array}$ \\
\hline
\end{tabular}

Abb. 4. Design der dreiarmigen ECOG-Studie.

7,1 Monate), was einer 27\%-igen Zunahme des medianen Überlebens entspricht. Dieser Unterschied zwischen beiden Studienarmen ist allerdings nicht signifikant, was sich laut Christophe Louvet, Hôpital Saint Antoine, Paris, durch die überraschend positiven Studienresultate, insbesondere auch des Kontrollarms erklären lässt: Erwartet worden waren Überlebenszeiten von 8 bzw. 6 Monaten. Das angestrebte Ziel - die 20\%-ige Steigerung der 8-Monats-Überlebensrate wurde zwar verfehlt (56,5 vs. 45,3\%); der Unterschied zwischen beiden Regimen zu diesem Zeitpunkt ist jedoch signifikant, betonte Louvet.

Über die Hälfte der Patienten erhielt nach Progression eine Second-line-Therapie. Louvet wies darauf hin, dass diese im Gemcitabin-Arm - entgegen dem Studienprotokoll - in fast drei Viertel der Fälle Platinderivate (einschließlich Oxaliplatin) beinhaltete. Dieser Faktor ist seiner Ansicht nach ein entscheidender Grund für den nur tendenziell ausgeprägten Überlebensvorteil der GEMOX-Kombination. Inwieweit die zusätzliche Gabe von Oxaliplatin oder die Applikation von Gemcitabin mit fixierter Dosierungsgeschwindigkeit zu der Überlebensverlängerung beiträgt, ist laut Louvet schwer auszumachen. Hedy Lee Kindeler, University of Chicago, kritisierte in ihrem Diskussionsbeitrag, dass die Gemcitabin-Regime in den beiden Studienarmen nicht vergleichbar waren. Diese Frage wird jedoch zur Zeit in einer ECOG-Studie der Phase III mit fast 800 Patienten geklärt: In 3 Armen werden Gemcitabin/ Oxaliplatin, die Monotherapie mit Gemcitabin als 30-minütiger Bolus und die Gemcitabin-Infusion verglichen (Abb. 4).

Laut Kindler ist es jetzt an der Zeit, auch beim fortgeschrittenen Pankreaskarzinom neue molekulare Substanzen in die Behandlung zu integrieren, da die alleinige konventionelle Chemotherapie den ungünstigen Verlauf bei diesem Tumor voraussichtlich nicht ändern wird. «Solche Ansätze werden die Therapie vermutlich mehr bereichern als die Erprobung weiterer Zytostatika-Doubletten», betonte sie. «Die moleku- laren Therapieansätze sollten zuammen mit der derzeit besten Chemotherapie geprüft werden, und das ist sicherlich die GEMOX-Kombination», so die Meinung von Louvet. Neben der höheren Effektivität zeichnet sich GEMOX auch durch eine gute Verträglichkeit aus; die Toxizität ist im Vergleich zur Gemcitabin-Monotherapie kaum erhöht.

\section{Oxaliplatin-Regime bei Magen- und Ösophaguskarzinom}

Schlecht ist die Prognose auch beim Magenkarzinom: Höchstens ein Drittel der betroffenen Patienten kann R0-reseziert werden; die langfristige Heilungsrate ist niedrig. Der palliativen Chemotherapie kommt daher große Bedeutung zu, weil sie die Lebensqualität im Vergleich zu alleinigen Supportivtherapie verbessert und eine Lebensverlängerung ermöglicht. Referenztherapie ist derzeit das ECF-Regime mit Epirubicin/Cisplatin/5-FU, mit dem Responseraten zwischen 40 und $45 \%$ und ein medianes Überleben von rund 9 Monaten erreicht werden. Aber: Das Regime gilt wegen der dauerhaften Versorgung der Patienten mit einer Pumpe als wenig patientenfreundlich, sodass nach therapeutischen Alternativen gesucht wird.

L. Cavanna und Mitarbeiter erprobten bei 35 Patienten mit fortgeschrittenem oder metastasierten Magenkrebs die Kombination von Oxaliplatin $\left(85 \mathrm{mg} / \mathrm{m}^{2}\right.$ i.v., Tag 1) mit einem 5-FU/FS-Infusionsregime [4]. Auf die Therapie sprachen 17 Patienten $(48,6 \%)$ an, einer von ihnen mit einer Komplettremission. Bei 10 weiteren Teilnehmern (28,6\%) wurde eine Tumorstabilisierung erreicht. Die hohe Aktivität ging mit einer nur leicht ausgeprägten Toxizität einher: Schwere Nebenwirkungen vom Grad 3/4 waren insgesamt selten. Am häufigsten wurden Neutropenien $(21,3 \%)$ und Thrombozytopenien registriert.

Beim inoperablen Ösophaguskarzinom wird derzeit eine Radiochemotherapie mit 4 Zyklen 5-FU/Cisplatin und einer Bestrahlung mit 50 Gy (RTOG-Regime) als Referenztherapie betrachtet, mit der eine Langzeit-Überlebensrate von 25\% erreicht wird. Giovani et al. erprobten in einer Phase-I-Studie ein Radiochemotherapie-Regime mit Ersatz von Cisplatin durch Oxaliplatin [11]. Die Dosisfindungsstudie an 33 Patienten zeigt, dass die Chemotherapie in einer dem FOLFOX4Regime entsprechenden Dosis sicher zusammen mit einer Bestrahlung von $50 \mathrm{~Gy}$ verabreicht werden kann. Bei den 28 evaluierbaren Patienten wurde eine Responserate von $61 \%$ erzielt: 3 Patienten sprachen komplett, 14 weitere partiell an. Die mediane Zeit bis zur Progression beträgt 5, die GesamtÜberlebenszeit 9 Monate. Jetzt ist geplant, die neue Kombination in einer kontrollierten Studie der Phase II/III mit dem RTOG-Referenzregime zu vergleichen.

Bericht: Dr. Katharina Arnheim, Berlin 


\section{Literatur}

1 André T, Boni C, Mounedji-Boudiaf L et al.: Oxaliplatin, Fluorouracil, and Leucovorin as Adjuvan Treatment for Colon Cancer. N Engl J Med 2004; 350:2343-2351.

2 André T, Maindrault-Goebel F, Mineur L et al.: Phase II study of an optimized 5FU/oxaliplatin strategy (OPTIMOX2) with celecoxib in metastatic colorectal cancer: A GERCOR study. Proc ASCO 2004;23:abstr 3554 (poster presentation)

3 Arkenau HT, Schmoll HJ, Kubicka S et al.: Phase III trial of capecitabine plus oxaliplatin (CAPOX) versus infusional 5-FU/LV plus oxaliplatin (FUFOX) in first-line metastatic colorectal cancer (MCRC): Interim safety analysis. Proc ASCO 2004; 23:abstr 3546 (poster presentation)

4 Cavanna L, Zaniboni A, Artioli F et al.: Oxaliplatin (OXA), 5-fluorouracil (5-FU) and leucovorin (LV) in patients with advanced or metastatic gastric cancer (A/MGC). Proc ASCO 2004;23:abstr 4068 (poster presentation).

5 Cassidy J, Scheithauer W, McKendrick W et al.: Capecitabine (X) vs bolus 5-FU/leucovorine (LV) as adjuvant therapy for colon cancer (the X-ACT study): Positive efficacy results of a phase III trial. Proc ASCO 2004;23:abstr 3509 (oral presentation).

6 De Gramont A, Cervantes A, André T et al.: OPTIMOX study: FOLFOX7/LV5FU2 compared to FOLFOX4 in patients with metastatic colorectal cancer. Proc ASCO 2004;23:abstr 3525 (poster presentation)

7 Figer A, Perez N, Carola E et al.: 5-fluorouracil, folinic acid and oxaliplatin (FOLFOX) in very old patients with metastatic colorectal cancer. Proc ASCO 2004:23:abstr 3571 (poster presentation).

8 Fisher GA, Kuo T, Cho CD et al.: A phase II study of IFOX (gefitinib with FOLFOX-4) in patients with metastastic colorectal cancer. Proc ASCO 2004;23:abstr 3514 (poster presentation)

9 Giacchetti S, Itzhaki M, Gruia G et al.: Long-term survival of patients with unresectable colorectal liver metastases following infusional chemotherapy with 5-fluorouracil, leucovorin and surgery Ann Oncol 1999;10:663-669.

10 Giacchetti S, Bjarnason G, Garufi C et al.: First line infusion of 5-fluorouracil, leucovorin and oxaliplatin for metastatic colorectal cancer: 4-day chronomodulated (FFL4-19) versus 2-day FOLFOX2. A multicenter randomized phase III trial of the chronotherapy group of the European Organization for Research and Treatment of Cancer (EORTC 05963). Proc ASCO 2004;23:abstr 3526 (poster presentation).
11 Giovani M, Conroy T, Francois E et al.: Phase I study of first line radiochemotherapy with oxaliplatin $(\mathrm{Ox}), 5$-fluoruracil (5-FU) and folinic acid in inoperable locally advanced or metastatic esophageal cancer. Proc ASCO 2004;23:abstr 4044 (poster presentation).

12 Goldberg RM, Morton RF, Sargent DJ et al.: N9741: Oxaliplatin (oxal) or CPT-11 + 5-fluorouracil (5-FU)/leucovorin (LV) or oxal + CPT-11 in advanced colorectal cancer (CRC): Updated efficacy and quality of life (QOL) data from an intergroup study. Proc ASCO 2003;22:abstr 1009 (oral presentation).

13 Goldberg RM, Sargent DJ, Morton RF et al.: N9741: FOLFOX (oxaliplatin (Oxal)/5-Fluoruracil (5-FU)/leucovorin (LV)) or reduced dosis R-IFL (CPT-11 + 5-FU/LV) in advanced colorectal cancer (CRC): Final efficacy data from an intergroup study. Proc ASCO 2004;23:abstr 3621 (poster presentation).

14 Grothey A, Jordan K, Kellner O et al.: Randomized phase II trial of capecitabine plus irinotecan (CapIri) vs capecitabine plus oxaliplatin (CapOx) as first-line-therapy of advanced colorectal cancer. Proc ASCO 2003;22:abstr 1011 (poster presentation).

15 Grothey A, Jordan K, Kellner O et al.: Capecitabine (Xeloda) plus either weekly irinotecan (CAPIRI) or weekly oxaliplatin (CAPOX) are active second-line protocols in patients with metastatic colorectal cancer (MCRC) after failure of firsttime combination therapy: Results of a randomized phase II study. Proc ASCO 2004;23:abstr 3534 (poster presentation).

16 Hickish T, Boni C, Navarro M et al.: FOLFOX 4 as adjuvant treatment for stage II colon cancer: Subpopulation data from the MOSAIC trial. Proc ASCO 2004;23:abstr 3619 (poster presentation)

17 Leonard GD, Fong Y, Jarnagin W et al.: Liver resection after hepatic arterial infusion (HAI) plus systemic oxaliplatin (Oxal) combination in pretreated patients with extensive unresectable colorectal liver metastases. Proc ASCO 2004;23:abstr 3542 (poster presentation).

18 Louvet C, André T, Lledo G et al.: Gemcitabine combined with oxaliplatin in advanced pancreatic adenocarcinoma: Final results of a GERCOR multicenter phase II study. J Clin Oncol 2002;20:15121518.
19 Louvet C, Labianca R, Hammel P et al.: GemOx (gemcitabine + oxaliplatin) versus gem (gemecitabine) in non resectable pancreatic adenocarcinoma: Final results of the GERCOR/GISCAID Intergroup Phase III. Proc ASCO 2004;23:abstr 4008 (oral presentation)

20 NIH consensus conference: Adjuvant therapy for patients with colon and rectal cancer. JAMA 1990; 264:1444-1450.

21 Saltz LR, Niedzwiecki D, Hollis D et al.: Irinotecan plus fluorouracil/leucovorin (IFL) versus fluorouracil/leucovorin alone (FL) in stage III colon cancer (intergroup trial CALBG C89803). Proc ASCO 2004;23:abstr 3500 (oral presentation).

22 Sargent DJ, Niedzwiecki D, O'Connell MJ et al: Recommendation for caution with irinotecan, fluoruracil, and leucovorin for colorectal cancer. N Engl J Med 2001;345:144-146.

23 Sargent DJ, Wieand S, Benedetti R et al.: Diseasefree survival (DFS) vs. overall survival (OS) as a primary endpoint for adjuvant colon cancer studies: Individual patient data from 12,195 patients on 15 randomized trials. Proc ASCO 2004;23:abstr 3502 (oral presentation).

24 Tabernero JM, van Cutsem E, Sastre J et al.: An international phase II study of cetuximab in combination with oxaliplatin/5-fluorouracil (5-FU)/folinic acid (FA) (FOLFOX-4) in the first-line treatment oft patients with metastatic colorectal cancer (CRC) expressing epidermal growth factor receptor (EGFR). Preliminary results. Proc ASCO 2004;23:abstr 3512 (poster presentation).

25 Tournigand C, Louvet C, Quinaux E et al FOLFIRI followed by FOLFOX versus FOLFOX followed by FOLFIRI in metastatic colorectal cancer (MRC): Final results of a phase III study. Proc ASCO 2002;22:abstr 494 (oral presentation).

26 Tournigand C, André T, Chirivella I et al.: 5-fluorouracil, folinic acid and oxaliplatin (FOLFOX) in poor prognosis. Proc ASCO 2004;23:abstr 3565 (poster presentation)

27 Welles L, Hochster H, Ramanathan R et al.: Preliminary results of a randomized study of the safety and tolerability of three oxaliplatin-based regimens as first-line treatment for advanced colorectal cancer (CRC) ('Tree' Study). Proc ASCO 2004;23: abstr 3537 (poster presentation). 